\title{
FIRST EXPERIMENTAL TESTS OF SHELS: A NEW HEAVY ION SEPARATOR AT THE JINR*
}

K. Rezynkina, K. Hauschild, A. Lopez-Martens

CSNSM, IN2P3-CNRS, UMR 8609, 91405 Orsay, France

O. Dorvaux, B. Gall, F. Déchery, H. Faure

IPHC-DRS/ULP, IN2P3-CNRS, 67037 Strasbourg, France

A.V. Yeremin, M.L. Chelnokov, V.I. Chepigin, A.V. Isaev I.N. Izosimov, D.E. Katrasev, A.N. Kuznetsov

A.A. Kuznetsova, O.N. Malyshev, A.G. Popeko, Y.A. Popov E.A. Sokol, A.I. Svirikhin

FLNR, JINR, 141980 Dubna, Russia

J. Piot, J. Rubert

GANIL, CEA/DSM-CNRS/IN2P3, BP 55027, 14076 Caen, France

(Received January 16, 2015)

A new Separator for Heavy ELement Spectroscopy (SHELS) has been recently installed at the U-400 accelerator at FLNR, JINR in Dubna, Russia. The details of the upgrade as well as the results from some of the commissioning runs are discussed. In particular, transmission tests with the ${ }^{208} \mathrm{~Pb}\left({ }^{40} \mathrm{Ar}, 2-3 n\right)^{245-6} \mathrm{Fm}$ reaction, as well as isomer spectroscopy results for ${ }^{210} \mathrm{Ra}$ produced in ${ }^{164} \mathrm{Dy}\left({ }^{50} \mathrm{Ti}, 3-5 n\right){ }^{209-11} \mathrm{Ra}$ reaction are presented.

DOI:10.5506/APhysPolB.46.623

PACS numbers: 29.30.-h, 23.20.Lv, 23.35. +g, 23.60.+e

\section{Introduction}

Due to the Coulomb repulsion between a large number of protons, the heaviest elements would not exist within the liquid-drop model. Transfermium nuclei (with $Z \geq 100$ ) are solely stabilized by quantum-mechanical

* Presented at the Zakopane Conference on Nuclear Physics "Extremes of the Nuclear Landscape", Zakopane, Poland, August 31-September 7, 2014. 
shell effects. One of the biggest challenges of nuclear physics is the search for the so-called island of stability - a cluster of super-heavy nuclei, that would be spherical and either stable or very long-lived. Predictions for the next magic numbers from different theoretical approaches vary widely: $Z=114$, 120 or 126 for the next proton shell and $N=172$ or 184 for the next neutron shell (see e.g. [1]). Some single-particle states involved in the stabilization of the heaviest elements are also active in the region of deformed transfermium nuclei around ${ }^{254}$ No. The cross section for the formation of these nuclei is many orders of magnitude higher than for $Z>110$, which makes them a unique laboratory to study the structure of nuclei in extreme conditions of charge and mass.

\section{The experimental setup}

\subsection{SHELS}

Transfermium nuclei are generally produced in fusion-evaporation reactions. Unreacted and scattered beam, as well as transfer, coulex and fusion-fission products, create an enormous background for these reactions. In order to eliminate this background, the separator SHELS is used. It was developed within the IN2P3-JINR Collaboration [2] and is a result of the upgrade of the VASSILISSA separator. This upgrade (see Fig. 1) consisted in a change from a kinetic energy separator to a velocity filter. The modernization had two main purposes: the extension of the region of accessibility for symmetric reactions which give evaporation residues (ER) of high electric rigidity $(\sim 10 \mathrm{MV})$ and the increase of the transmission for very asymmetric reactions (e.g. $\mathrm{Ne}$ on $\mathrm{Pu})$.



Fig. 1. Schematic view of the SHELS (top) and the VASSILISSA (bottom) separators. 


\subsection{GABRIELA}

The ERs of interest selected by the separator are transported to the detection system GABRIELA (Gamma Alpha Beta Recoil Investigations with the ELectromagnetic Analyser) [3, 4], where they pass through a time-offlight detector and are implanted into a Double-sided Silicon Strip Detector (DSSD). The DSSD is surrounded by a box of silicon strip detectors and an array of Ge detectors. The setup is sensitive to many types of decays: fission, $\alpha$ - and $\beta$-decay, as well as de-excitations via $\gamma$-rays and internal conversion electrons (ICE) and subsequent atomic processes.

The detection system was also upgraded: the size of the DSSD was increased from $58 \times 58 \mathrm{~mm}^{2}$ to $100 \times 100 \mathrm{~mm}^{2}$, which is essential for the asymmetrical reactions. The granularity of the silicon detectors was also increased to allow for longer correlation times. The Ge array is currently undergoing an upgrade in order to increase the efficiency, peak-to-total and granularity.

\section{The commissioning of SHELS}

$$
\text { 3.1. }{ }^{246} \mathrm{Fm}
$$

One of the reactions used for the commissioning of the upgraded setup was the following: ${ }^{208} \mathrm{~Pb}\left({ }^{40} \mathrm{Ar}, 2-3 n\right){ }^{245-6} \mathrm{Fm} .{ }^{245} \mathrm{Fm} \alpha$-decays, and ${ }^{246} \mathrm{Fm}$ has a $93.20 \% \alpha$-branch and $6.80 \%$ spontaneous fission (SF) branch [5]. The excitation functions for this reaction are given in Fig. 2 (a). Figure 2 (b) contains the spectra of $\alpha$ decay of both ${ }^{245} \mathrm{Fm}$ and ${ }^{246} \mathrm{Fm}$. The production ratio of these two nuclei was used for the precise definition of the beam energy. From the number of observed decay events, and given a measured beam dose on target, a preliminary value of $40 \%$ was obtained for the transmission of the ERs. This indicates a factor of two improvement of the transmission after the upgrade. The transmission measured for a neon on gold reaction with a $58 \times 58 \mathrm{~mm}^{2}$ DSSD has also yielded over a factor of two (and this result will further improve with a $\left.100 \times 100 \mathrm{~mm}^{2} \mathrm{DSSD}\right)$.


Fig. 2. (a) Excitation functions for the ${ }^{208} \mathrm{~Pb}\left({ }^{40} \mathrm{Ar}, x n\right)$ reaction taken from [6]; (b) fit to the $\alpha$-decay lines of ${ }^{245} \mathrm{Fm}$ and ${ }^{246} \mathrm{Fm}$ and corresponding energies. 


\section{2. ${ }^{210} R a$}

Another commissioning reaction was ${ }^{164} \mathrm{Dy}\left({ }^{50} \mathrm{Ti}, 3-5 n\right){ }^{209-11} \mathrm{Ra}$, where the newly developed Ti beam [7] was first used at the JINR. ${ }^{210} \mathrm{Ra}$ has a $\mathrm{T}_{1 / 2}=2.1 \mu \mathrm{s}$ isomeric state which decays through a cascade of $96.5,578$, 602, 604, 751 and $775 \mathrm{keV}$ transitions. From the measured spectra (see Fig. 3), we have deduced the conversion coefficients for all these transitions ( $\alpha_{\text {tot }}$ for $96.5 \mathrm{keV}$, and $\alpha_{K}$ and $\alpha_{L}$ for the rest), and assigned all of them to have E2 nature. Thus, we confirm the level scheme proposed in [8]. This is the first direct measurement of these conversion coefficients.
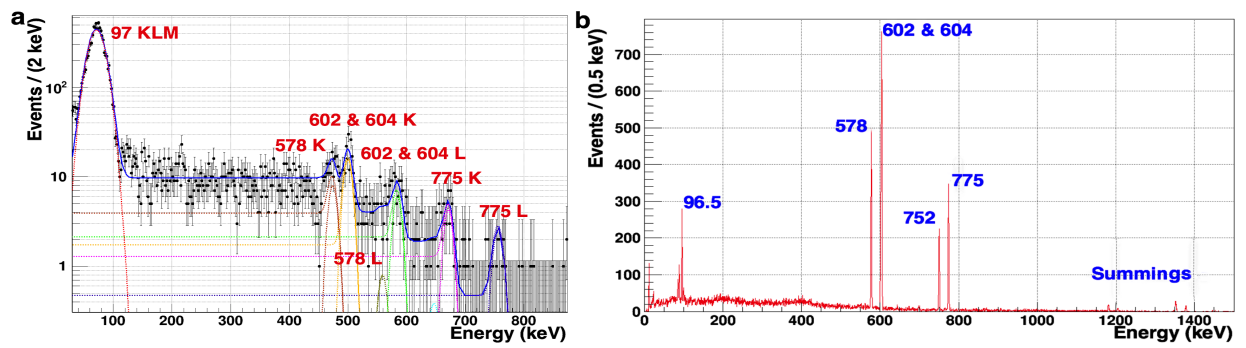

Fig. 3. The ICE (a) and $\gamma$ - and X-ray (b) spectra for the transitions depopulating the $2.1 \mu$ s isomer in ${ }^{210} \mathrm{Ra}$.

\section{Conclusions and outlook}

The commissioning of SHELS has shown the expected performance of the setup after the modernization. The transmission was increased by a factor of two for Ar, Ca and Ti beams, and more than that for the asymmetrical reactions. A new detector chamber, as well as Ge array and BGO shields are now under development and will be put in operation soon. The first experimental campaign is planned for spring 2015, producing No isotopes.

\section{REFERENCES}

[1] M. Bender et al., Phys. Rev. C60, 034304 (1999).

[2] SHELS: ANR-06-BLAN-0034-01, 2006-2011.

[3] CLODETTE: ANR-12-BS05-0013-02, 2013-2017.

[4] K. Hauschild et al., Nucl. Instrum. Methods Phys. Res. A560, 388 (2006).

[5] NNDC, http://www.nndc.bnl.gov/chart/

[6] A.I. Svirikhin et al., Eur. Phys. J. A44, 393 (2010).

[7] J. Rubert et al., Nucl. Instrum. Methods Phys. Res. B276, 33 (2012).

[8] J.J. Ressler et al., Phys. Rev. C69, 034331 (2004). 